\title{
DETERMINATION OF INSPECTION SCHEDULES OF EQUIPMENT BY VARIATIONAL METHOD
}

\author{
SUSUMU OKUMURA
}

Received 4 January 2006; Accepted 24 March 2006

Maintained equipment can be subject to random failures. If failures are evident, they can be detected immediately when they occur. On the other hand, hidden failures that are not revealed during the performance of regular duties may be detected by inspection process. If concerned equipment has hidden failures, loss is assumed to incur from the moment its failure until the time when it is detected. Optimal inspection schedule should be considered when inspection is costly. In this study, optimal inspection schedules for equipment are derived by the variational method employing the inspection density function. For the case in which the time-to-failure distribution of equipment is given, a conditional equation that optimal inspection schedules should satisfy is derived. Furthermore, when the time-to-failure distribution is unknown, an ordinary differential equation that optimal inspection schedules should satisfy is obtained. Optimal inspection schedules in a closed form and numerical examples are shown for some potential loss rate functions.

Copyright (C 2006 Susumu Okumura. This is an open access article distributed under the Creative Commons Attribution License, which permits unrestricted use, distribution, and reproduction in any medium, provided the original work is properly cited.

\section{Introduction}

Inspection is one of the means to confirm whether maintained equipment operates normally, or whether it works correctly when required for operation, and identify deterioration level of equipment. Cost of inspection increases when the inspection interval is shortened since inspection is a cost element. On the contrary, loss or risk caused by failures will increase when the inspection interval is lengthened. Therefore, an appropriate setting of inspection schedules is important with consideration of a tradeoff between inspection cost and loss due to failure of equipment.

If equipment deteriorates gradually and the operational condition of equipment can be identified by inspection process based on condition monitoring techniques using measurement of vibration or wear, the deterioration trend plot can be useful for determining the optimal inspection schedule [10]. On the other hand, if equipment has a nature of sudden malfunction such as cracks of materials, frequent inspection will be effective since 
deterioration of maintained equipment is difficult to predict. When the reliability function of the time to failure of equipment is identified, the optimal inspection schedule can be determined based on a mathematical inspection model employing the reliability function. Barlow and Proschan [1] formulated an inspection model, in which the time-tofailure distribution is known, and obtained the recursive relation between two successive inspection times. The literature on maintenance and replacement models was surveyed $[2,9,11,12]$. Leung [7] discussed basic models for the detection of system failures by inspection.

Optimal inspection schedules for a single-unit system whose time to failure is described by a probability distribution were derived based on the variational method [5-7]. Time-to-failure distribution may be difficult to identify when few data for the distribution are available. Therefore, Keller [6] and Leung [7] examined optimal inspection schedules under the assumption that a probability distribution of the time to failure is unknown. However, loss caused by a failure is limited to a simple linear loss rate function [5-7]. Okumura and Okino [8] studied optimal inspection schedules when failed equipment has a general type of loss rate function.

In this study, a method for determining optimal inspection schedules is discussed, in which failures of equipment are detected only by inspection. The inspection density function proposed by Keller [6], which generates inspection schedules, is employed in a minimization problem of the incurred cost per cycle. An optimal inspection density function is derived by the variational method. The optimal inspection schedules in a closed form are shown for some potential failure distributions and loss rate functions. Then, optimal inspection density functions, optimal sequences of inspection times, and the conditions that inspection is effective are obtained explicitly for the case that failure distributions are unknown.

\section{Formulation and optimal inspection density function}

2.1. Assumptions. The following assumptions are made.

(1) Equipment is a single-unit system.

(2) When a system fails, the failed state is detected only by inspection with probability 1 .

(3) Inspection is conducted at time $t_{k}(k=1,2, \ldots)$ with negligible time for an inspection.

(4) A probability density function and a cumulative distribution function of the time to failure of a system exists, which are denoted by $f(t)$ and $F(t)(t \geq 0)$, respectively. A failure rate function is denoted by $\lambda(t)=f(t) / \bar{F}(t)(t \geq 0)$, where $\bar{F}(t) \equiv 1-F(t)$.

(5) The time-to-failure distribution is not influenced by inspection.

(6) Loss, which is characterized by a loss rate function $L(t)$, is incurred from the moment the system fails until the time when it is detected.

(7) A continuous inspection density function $n(t)$ exists, which gives the approximate number of inspections per unit time [6]. Herewith, the inspection interval is approximately given by $1 / n(t)$. If $n(t)$ is identified, $t_{k}$ are determined since we 
have the relation $\int_{0}^{t_{k}} n(t), d t=k[5]$. Here, inspection time is given by

$$
t_{k}=N^{-1}(N(0)+k), \quad k=1,2, \ldots,
$$

where $N(t)$ is the primitive function of $n(t)$.

(8) An inspection cost is denoted by $c$.

2.2. Expected incurred cost. The expected incurred inspection cost until the failure of a system is detected is given by

$$
c \int_{0}^{\infty}\left(\int_{0}^{t} n(x) d x\right) f(t) d t
$$

Equation (2.2) is transformed to

$$
c \int_{0}^{\infty} \bar{F}(t) n(t) d t
$$

since $F(0)=0$ and $F(\infty)=1$. Then, the sum of approximate loss caused by failure of a system and replacement cost is written as

$$
\int_{0}^{\infty}\left(n(t) \int_{0}^{1 / n(t)} L(x) d x\right) f(t) d t .
$$

Therefore, the sum of the expected costs $E[C]$, whose elements are inspection cost and loss cost, is approximately given by

$$
E[C]=c \int_{0}^{\infty} \bar{F}(t) n(t) d t+\int_{0}^{\infty}\left(n(t) \int_{0}^{1 / n(t)} L(x) d x\right) f(t) d t,
$$

which arises in a sequence of time: an as-good-as-new state that begins at $t=0$, a failed state whose time of occurrence is characterized by $F(t)$, and duration of the failed state until the failure is detected by inspection.

2.3. Optimal inspection density function when time-to-failure distribution is given. The Euler equation is derived in order to find $n(t)$ which minimizes (2.5):

$$
\frac{1}{n(t)} L\left(\frac{1}{n(t)}\right)-\int_{0}^{1 / n(t)} L(x) d x=\frac{c}{\lambda(t)} .
$$

Then, we solve nonlinear equations for $n(t)$, which are derived by substituting potential $L(t)$ into (2.6). Here, optimal inspection density functions are shown for three types of $L(t)$.

(1) $L(t)=c_{1} t^{p}+c_{2}\left(p, c_{1}, c_{2}\right.$ : positive constant).

Equation (2.6) gives

$$
n_{1}^{*}(t)=\left(\frac{c_{1} p}{c(p+1)} \lambda(t)\right)^{1 /(p+1)}
$$




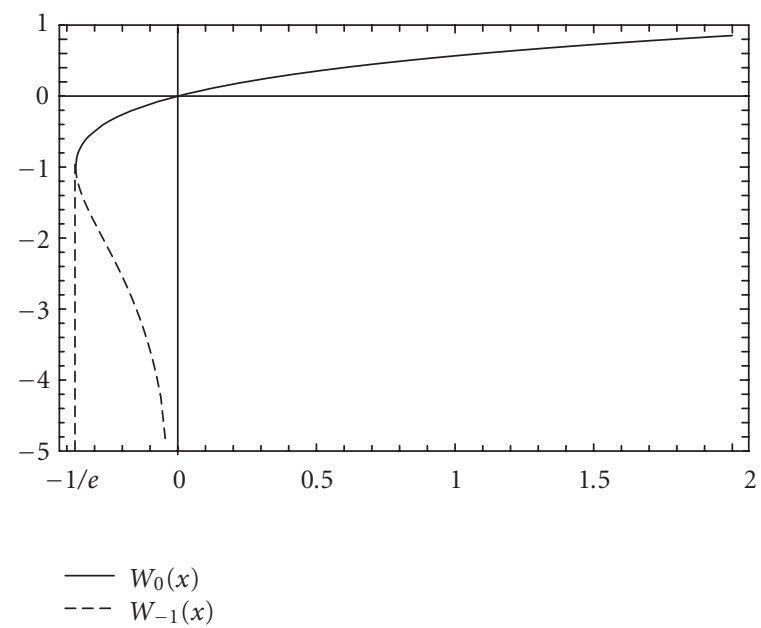

Figure 2.1. The real values of the Lambert $W$ function: the solid line shows $W_{0}(x)$ and the dashed line $W_{-1}(x)$.

(2) $L(t)=c_{1} t^{2}+c_{2} t+c_{3}\left(c_{1}, c_{2}, c_{3}:\right.$ positive constant $)$.

The following optimal inspection density function is obtained:

$$
n_{2}^{*}(t)=\frac{k_{1} \lambda(t)}{G(\lambda(t))}+G(\lambda(t))
$$

where $G(x)=\left\{x\left(k_{2}+\left(k_{2}^{2}-k_{1}^{3} x\right)^{1 / 2}\right)\right\}^{1 / 3}, k_{1}=c_{2} / 6 c, k_{2}=c_{1} / 3 c$.

(3) $L(t)=c_{1}\left(\exp \left[c_{2} t\right]-1\right)+c_{3}\left(c_{1}, c_{2}, c_{3}\right.$ : positive constant $)$.

From (2.6),

$$
n_{3}^{*}(t)=\left[\frac{1}{c_{2}}\left\{1+W\left(\frac{1}{e}\left(\frac{c c_{2}}{c_{1} \lambda(t)}-1\right)\right)\right\}\right]^{-1}
$$

is derived, where $W(\cdot)$ is the Lambert $W$ function as shown in Figure 2.1, which is the complex-valued function that satisfies $W(x) \exp [W(x)]=x$ for all $x \in \mathbb{C}$.

Note that (2.7)-(2.9) are irrelevant to absolute terms $c_{2}$ and $c_{3}$ in $L(t)$. The increasing and decreasing properties of $n_{i}^{*}(t)(i=1,2$, and 3$)$ correspond to those of $\lambda(t)$ at every $L(t)$.

Remark 2.1. Keller [6] derived the Euler equation from the functional in which the righthand side of the first term in (2.5) is given by (2.2), and under the conditions that $L(t)$ is linear, that is,

$$
c \int_{0}^{\infty}\left(\int_{0}^{t} n(x) d x\right) f(t) d t+\int_{0}^{\infty} L\left(\frac{1}{2 n(t)}\right) f(t) d t .
$$


The Euler equation for (2.10) yields

$$
\frac{d}{d t}\left\{f(t) \frac{\partial}{\partial n(t)} L\left(\frac{1}{2 n(t)}\right)\right\}=c f(t)
$$

Then, the following equation for $n(t)$ is derived:

$$
\frac{1}{\{n(t)\}^{2}} L^{\prime}\left(\frac{1}{2 n(t)}\right)=\frac{2 c(u-F(t))}{f(t)}
$$

where $u$ is an integration constant. When $L(t)=c_{1} t$, after finding $n(t)$ which satisfies (2.12) and substituting it into (2.10), a minimization problem of $u$ is obtained. Finally, the optimal inspection density function yields

$$
n^{*}(t)=\left\{\frac{c_{1}}{2 c} \lambda(t)\right\}^{1 / 2}
$$

On the other hand, the functional derived by Kaio and Osaki [5] and Leung [7] was based on the condition that $L(t)=c_{1} t$ :

$$
c \int_{0}^{\infty} \bar{F}(t) n(t) d t+\frac{c_{1}}{2} \int_{0}^{\infty} \frac{f(t)}{n(t)} d t .
$$

From the Euler equation,

$$
\{n(t)\}^{2}=\frac{c_{1}}{2 c} \lambda(t)
$$

which minimizes (2.14) for $n(t)$, is obtained. Then, (2.13) is derived.

Note that (2.13) in [5-7] corresponds to the result of $p=1$ in (2.7).

Remark 2.2. When $L(t)$ is approximated by a second-order equation, we obtain

$$
\int_{0}^{\infty}\left(n(t) \int_{0}^{1 / n(t)} L(x) d x\right) f(t) d t=\int_{0}^{\infty}\left\{L\left(\frac{1}{2 n(t)}\right)+\frac{1}{24\{n(t)\}^{2}} L^{\prime \prime}\left(\frac{1}{2 n(t)}\right)\right\} f(t) d t .
$$

Then, the Euler equation for finding $n(t)$ that minimizes (2.5) satisfies

$$
\frac{1}{2\{n(t)\}^{2}} L^{\prime}\left(\frac{1}{2 n(t)}\right)+\frac{1}{12\{n(t)\}^{3}} L^{\prime \prime}\left(\frac{1}{2 n(t)}\right)+\frac{1}{48\{n(t)\}^{4}} L^{(3)}\left(\frac{1}{2 n(t)}\right)=\frac{c}{\lambda(t)} \text {. }
$$

Equation (2.8) can be obtained by solving the equation for $n(t)$ after substituting $L(t)=$ $c_{1} t^{2}+c_{2} t+c_{3}$ into $(2.17)$.

Sufficient condition. Equation (2.5) is written as

$$
E[C]=\int_{0}^{\infty} H(t, n(t)) d t
$$


in which the Legendre-Hadamard condition [3] yields $H_{n^{\prime} n^{\prime}}=0$ and $H_{n n^{\prime}}=0$. When $H(t)+\alpha \xi(t)$ is the admissible function, the second variation of $E[C]$ follows

$$
\begin{aligned}
\delta^{2} E[C] & =\int_{0}^{\infty}\{\xi(t)\}^{2} H_{n n} d t \\
& =\int_{0}^{\infty} \frac{\{\xi(t)\}^{2} f(t)}{\{n(t)\}^{3}} L^{\prime}\left(\frac{1}{n(t)}\right) d t .
\end{aligned}
$$

Therefore, if $L(t)$ is nondecreasing, the second variation satisfies $\delta^{2} E[C]>0$, which is apparent in every loss ratio function treated in this paper.

2.4. Optimal inspection density function when time-to-failure distribution is unknown. When $\lambda(t)$ is given, optimal inspection density function $n^{*}(t)$ which minimizes $E[C]$ is derived from (2.6). If $\lambda(t)$ or $F(t)$ is unknown, a minimax solution, that is, $\max _{F(t)} \min _{n(t)} E[C]$, is considered, by which conservative inspection schedules can be obtained.

From (2.6) we can see that the optimal inspection density function is a function of $\lambda(t)$, that is, $n^{*}(t)=q(\lambda(t))$, then

$$
\max _{F(t)} \min _{n(t)} E[C] \Longleftrightarrow \max _{\lambda(t)} \min _{n(t)} E[C]
$$

Since we have the relations $F(t)=1-\exp \left[-\int_{0}^{t} \lambda(t) d t\right]$ and $f(t)=\lambda(t) \exp \left[-\int_{0}^{t} \lambda(t) d t\right]$, the Euler equation yields

$$
\begin{aligned}
& \left\{\left(\lambda^{2}-2 \lambda^{\prime}\right) q^{\prime}-\lambda \lambda^{\prime} q^{\prime \prime}\right\}\left\{q \int_{0}^{1 / q} L(x) d x-L\left(\frac{1}{q}\right)\right\} \\
& -c q\left(q-\lambda q^{\prime}+\lambda^{\prime} q^{\prime \prime}\right)-\lambda \lambda^{\prime}\left(\frac{q^{\prime}}{q}\right)^{2} L^{\prime}\left(\frac{1}{q}\right)=0,
\end{aligned}
$$

where $q$ and $\lambda$ are abbreviations of $q(\lambda(t))$ and $\lambda(t)$, respectively.

When $L(t)=c_{1} t^{p}+c_{2}$,

$$
q_{1}(x)=\left(\frac{c_{1} p}{c(p+1)} x\right)^{1 /(p+1)}
$$

from (2.7). Then (2.21) becomes

$$
\lambda_{1}^{\prime}(t)-(p+1)\left\{\lambda_{1}(t)\right\}^{2}=0
$$

If $\lambda_{1}(0)=a$ is assumed to be an integration constant,

$$
\lambda_{1}(t)=\frac{a}{1-(p+1) a t}
$$


is derived from (2.23). Then, we have

$$
\begin{aligned}
& n_{1}^{* *}(t)= q_{1}\left(\lambda_{1}(t)\right) \\
&=\left(\frac{c_{1} p}{c(p+1)} \cdot \frac{a}{1-(p+1) a t}\right)^{1 /(p+1)} \quad \text { for } 0 \leq t<\frac{1}{a(p+1)}, \\
& \max _{\lambda(t)} \min _{n(t)} E[C]=\left\{\frac{c_{1}}{p+1}\left(\frac{c}{a p}\right)^{p}\right\}^{1 /(p+1)}+c_{2} .
\end{aligned}
$$

When $L(t)=c_{1} t^{2}+c_{2} t+c_{3},(2.21)$ becomes

$$
\begin{aligned}
Z_{21}^{5}- & \left(\lambda_{2} Z_{22}-\lambda_{2}^{\prime} Z_{23}\right) Z_{21}^{4}+\left(3 k_{1} Z_{21}+2 k_{2}\right)\left\{\left(\lambda_{2}^{2}-2 \lambda_{2}^{\prime}\right) Z_{22}-\lambda_{2} \lambda_{2}^{\prime} Z_{23}\right\} Z_{21} \\
& +6 \lambda_{2} \lambda_{2}^{\prime}\left(k_{1} Z_{21}+k_{2}\right) Z_{22}^{2}=0
\end{aligned}
$$

where

$$
\begin{gathered}
\lambda_{2}:=\lambda_{2}(t), \quad q_{2}:=G\left(\lambda_{2}\right), \quad Z_{21}:=\frac{k_{1} \lambda_{2}}{q_{2}}+q_{2}, \\
Z_{22}:=\frac{k_{1}\left(1-\lambda_{2} q_{2}^{\prime} / q_{2}\right)}{q_{2}}+q_{2}^{\prime}, \\
Z_{23}:=\frac{2 k_{1} \lambda_{2}\left(q_{2}^{\prime}\right)^{2}}{q_{2}^{3}}-\frac{k_{1}\left(\lambda_{2} q_{2}^{\prime \prime}+2 q_{2}^{\prime}\right)}{q_{2}^{2}}+q_{2}^{\prime \prime} .
\end{gathered}
$$

For $L(t)=c_{1}\left(\exp \left[c_{2} t\right]-1\right)+c_{3}$

$$
\begin{aligned}
\lambda_{3}^{2} Z_{31} & \left(Z_{32}+1\right)^{2}\left\{Z_{31}+\left(Z_{32}^{2}+Z_{32}+1\right) \exp \left[Z_{32}+1\right]\right\} \\
- & \lambda_{3}^{\prime} Z_{32}\left(Z_{31}+1\right)\left\{2 Z_{31}+\left(Z_{32}+1\right) \exp \left[Z_{32}+1\right]\right\}=0
\end{aligned}
$$

is derived from (2.21), where

$$
\lambda_{3}:=\lambda_{3}(t), \quad Z_{31}:=\frac{c c_{2}}{c_{1} \lambda_{3}}-1, \quad Z_{32}:=W\left(\frac{Z_{31}}{e}\right)
$$

A numerical method is appropriate for solving (2.28) and (2.30).

Remark 2.3. Keller [6] and Leung [7] obtained their minimax solution by maximizing the expected cost with regard to $F(t)$. Their solution is a special case of (2.26) and (2.27) derived in this paper, by substituting $p=1, a=\lambda / 2$ (constant), and $c_{2}=0$ into the equations.

Sufficient condition. When $L(t)=c_{1} t^{p}+c_{2}$, the extremal resulting from the Euler equation is (2.24) under the conditions that $\lambda(0)=0$ and $\lambda(1 / a(p+1))=\infty$. The extremal has a proper field but does not have a central field in the range of $\{t \mid 0 \leq t \leq 1 / a(p+1)\}$. The 
Table 3.1. Summary of results obtained when time-to-failure distribution is given. $L_{1}: L(t)=c_{1} t^{p}+$ $c_{2}, L_{2}: L(t)=c_{1} t^{2}+c_{2} t+c_{3}, L_{3}: L(t)=c_{1}\left(\exp \left[c_{2} t\right]-1\right)+c_{3} ; C_{1}$ : number of inspections, $C_{2}$ : optimal inspection time, $C_{3}$ : condition that the number of inspections is $m(m=1,2, \ldots), C_{4}$ : condition that inspection is effective; $\bigcirc$ : explicit form solution, $\times$ : no explicit form solution.

\begin{tabular}{l|cccc|cccc|cccc}
\hline Loss rate & \multicolumn{6}{|c|}{$L_{1}$} & \multicolumn{5}{|c}{$L_{2}$} & \multicolumn{5}{c}{$L_{3}$} \\
\hline Classification & $C_{1}$ & $C_{2}$ & $C_{3}$ & $C_{4}$ & $C_{1}$ & $C_{2}$ & $C_{3}$ & $C_{4}$ & $C_{1}$ & $C_{2}$ & $C_{3}$ & $C_{4}$ \\
\hline Weibull distribution & $\bigcirc$ & $\bigcirc$ & $\bigcirc$ & $\bigcirc$ & $\times$ & $\times$ & $\times$ & $\times$ & $\times$ & $\times$ & $\times$ & $\times$ \\
Exponential distribution & $\bigcirc$ & $\bigcirc$ & $\bigcirc$ & $\bigcirc$ & $\bigcirc$ & $\bigcirc$ & $\bigcirc$ & $\bigcirc$ & $\bigcirc$ & $\bigcirc$ & $\times$ & $\times$ \\
Uniform distribution & $\bigcirc$ & $\bigcirc$ & $\bigcirc$ & $\bigcirc$ & $\times$ & $\times$ & $\times$ & $\times$ & $\bigcirc$ & $\bigcirc$ & $\bigcirc$ & $\bigcirc$ \\
\hline
\end{tabular}

Legendre-Hadamard condition for arbitrary $\lambda(t) \neq 0$ is negative:

$$
-\frac{c \exp \left[-\int_{0}^{t} \lambda(x) d x\right]}{(p+1)\{\lambda(t)\}^{2}}\left(\frac{c_{1} p}{c(p+1)} \lambda(t)\right)^{1 /(p+1)}<0 .
$$

When $\lambda(t)=0$, the Legendre-Hadamard condition has singularity. Therefore, (2.24) is a weak maximizer.

When $L(t)=c_{1} t^{2}+c_{2} t+c_{3}$ and $L(t)=c_{1}\left(\exp \left[c_{2} t\right]-1\right)+c_{3}$, the Legendre-Hadamard condition is negative for arbitrary $\lambda(t)$. However, optimal $\lambda(t)$ cannot be obtained explicitly; therefore, it is difficult to check the Jacobi condition for both loss rate functions.

\section{Some explicit results}

3.1. When time-to-failure distribution is given. The Weibull distribution, a negative exponential distribution, and a uniform distribution are considered for the solution of optimal inspection schedules. Equations in a closed form for (1) the number of inspections, (2) optimal inspection time, (3) the condition that the number of inspections is $m$ $(m=1,2, \ldots)$, and (4) the condition that inspection is effective are derived. The obtained equations are arranged in Table 3.1.

3.1.1. Failure distribution: the Weibull distribution. When $L(t)=c_{1} t^{p}+c_{2}$ and the time to failure is provided by the Weibull distribution $\lambda(t)=\beta / \eta \cdot(t / \eta)^{\beta-1}$, the following is obtained in the range of $\{t \mid F(t) \leq 1-\varepsilon\} \Longleftrightarrow\left\{t \mid 0 \leq t \leq \eta\left(\ln \varepsilon^{-1}\right)^{1 / \beta}\right\}$, where $\varepsilon$ is a small positive constant.

(i) Number of inspections:

$$
\begin{aligned}
n_{I} & =\left\lfloor\int_{0}^{\eta\left(\ln \varepsilon^{-1}\right)^{1 / \beta}} n_{1}^{*}(t) d t\right\rfloor \\
& =\left\lfloor\frac{\eta(p+1)}{(p+\beta)}\left(\frac{c_{1} p}{c(p+1)} \cdot \frac{\beta}{\eta}\left(\ln \varepsilon^{-1}\right)^{p / \beta+1}\right)^{1 /(p+1)}\right\rfloor,
\end{aligned}
$$

where $\lfloor x\rfloor$ denotes the greatest integer less than or equal to $x$. 
(ii) Optimal inspection time:

$$
t_{k}=\left\{\eta^{\beta-1}\left(\frac{c_{1} p}{c(p+1)} \cdot \frac{\beta}{\eta}\right)^{-1}\left(\frac{k(p+\beta)}{p+1}\right)^{p+1}\right\}^{1 /(p+\beta)}, k=1, \ldots, n_{I} .
$$

(iii) Condition that the number of inspections is $m$ :

$$
r_{1}(m+1)<\frac{c_{1}}{c} \leq r_{1}(m)
$$

where

$$
r_{1}(m)=\left(\frac{m(p+\beta)}{\eta(p+1)}\right)^{p+1}\left(\frac{p}{p+1} \cdot \frac{\beta}{\eta}\left(\ln \varepsilon^{-1}\right)^{p / \beta+1}\right)^{-1} .
$$

(iv) Condition that inspection is effective, that is, $n_{I} \geq 1$ :

$$
\frac{c_{1}}{c} \geq r_{1}(1)
$$

For $L(t)=c_{1} t^{2}+c_{2} t+c_{3}$ and $L(t)=c_{1}\left(\exp \left[c_{2} t\right]-1\right)+c_{3}$, when $\lambda(t)$ is the Weibull distribution, it is difficult to derive explicit results. However, if the time-to-failure distribution is a negative exponential, that is, $\beta=1, \lambda(t)=1 / \lambda$, it is possible to derive some equations in a closed form.

3.1.2. Failure distribution: negative exponential distribution. When $L(t)=c_{1} t^{p}+c_{2}$, the results are the same as those substituted $\beta=1$ into (3.2), (3.3), and (3.5):

$$
\begin{gathered}
n_{I}=\left\lfloor\eta\left(\ln \varepsilon^{-1}\right)\left(\frac{c_{1} p}{\eta c(p+1)}\right)^{1 /(p+1)}\right\rfloor, \\
t_{k}=k\left(\frac{\eta c(p+1)}{c_{1} p}\right)^{p+1}, \\
r_{1}(m)=\frac{\eta(p+1)}{p}\left(\frac{m}{\eta \ln \varepsilon^{-1}}\right)^{p+1} .
\end{gathered}
$$

When $L(t)=c_{1} t^{2}+c_{2} t+c_{3}$ and $\lambda(t)=1 / \eta$, the following equations are derived.

(i) Number of inspections:

$$
\begin{aligned}
n_{I} & =\left\lfloor\int_{0}^{\eta \ln \varepsilon^{-1}} n_{2}^{*}(t) d t\right\rfloor \\
& =\left\lfloor\frac{\left\{k_{1}+\eta\{G(1 / \eta)\}^{2}\right\} \ln \varepsilon^{-1}}{G(1 / \eta)}\right\rfloor .
\end{aligned}
$$

(ii) Optimal inspection time:

$$
t_{k}=\frac{k}{G(1 / \eta)+k_{1} / \eta G(1 / \eta)}, \quad k=1, \ldots, n_{I} .
$$


(iii) Condition that the number of inspections is $m$ :

$$
r_{2}(m) \leq \eta<r_{2}(m+1)
$$

where

$$
r_{2}(m)=\frac{m\left\{\left(9 k_{1}^{2} \ln \varepsilon^{-1}+8 k_{2} m\right)^{1 / 2}-3 k_{1}\left(\ln \varepsilon^{-1}\right)^{1 / 2}\right\}}{4 k_{2}\left(\ln \varepsilon^{-1}\right)^{3 / 2}} .
$$

(iv) Condition that inspection is effective:

$$
\eta \geq r_{2}(1)
$$

If $L(t)=c_{1}\left(\exp \left[c_{2} t\right]-1\right)+c_{3}$, the following are obtained.

(i) Number of inspections:

$$
\begin{aligned}
n_{I} & =\left\lfloor\int_{0}^{\eta \ln \varepsilon^{-1}} n_{3}^{*}(t) d t\right\rfloor \\
& =\left\lfloor\left[\frac{1}{c_{2} \eta \ln \varepsilon^{-1}}\left\{1+W\left(\frac{1}{e}\left(\frac{c c_{2} \eta}{c_{1}}-1\right)\right)\right\}\right]^{-1}\right\rfloor .
\end{aligned}
$$

(ii) Optimal inspection time:

$$
t_{k}=\frac{k}{c_{2}}\left\{1+W\left(\frac{1}{e}\left(\frac{c c_{2} \eta}{c_{1}}-1\right)\right)\right\}, \quad k=1, \ldots, n_{I} .
$$

The condition that the number of inspections is $m$ and the condition that inspection is effective cannot be easily derived in a closed form.

3.1.3. Failure distribution: uniform distribution. When $L(t)=c_{1} t^{p}+c_{2}$ and time-to-failure distribution is provided with a uniform distribution in the range of $[0, T]$, the following results are obtained.

(i) Number of inspections:

$$
\begin{aligned}
n_{I} & =\left\lfloor\int_{0}^{T} n_{1}^{*}(t) d t\right\rfloor \\
& =\left\lfloor\left\{\frac{c_{1}}{c} T^{p}\left(\frac{p}{p+1}\right)^{p+2}\right\}^{1 /(p+1)}\right\rfloor .
\end{aligned}
$$

(ii) Optimal inspection time:

$$
t_{k}=T-\left[T^{p /(p+1)}-k\left\{\frac{c}{c_{1}}\left(\frac{p+1}{p}\right)^{p+2}\right\}^{1 /(p+1)}\right]^{(p+1) / p}, k=1, \ldots, n_{I} .
$$

(iii) Condition that the number of inspections is $m$ :

$$
r_{3}(m) \leq T<r_{3}(m+1)
$$


where

$$
r_{3}(m)=\left\{\frac{c}{c_{1}}\left(\frac{p+1}{p}\right)^{p+2} m^{p+1}\right\}^{1 / p} .
$$

(iv) Condition that inspection is effective:

$$
T \geq r_{3}(1)
$$

When $L(t)=c_{1} t^{2}+c_{2} t+c_{3}$, every result is difficult to write in a closed form; however, when $L(t)=c_{1}\left(\exp \left[c_{2} t\right]-1\right)+c_{3}$, the following are derived.

(i) Number of inspections:

$$
\begin{aligned}
n_{I} & =\left\lfloor\int_{0}^{T} n_{3}^{*}(t) d t\right\rfloor \\
& =\left\lfloor\frac{c_{1}}{c}\left\{\exp \left[1+W\left(\frac{c c_{2} T-c_{1}}{e c_{1}}\right)\right]-1\right\}\right\rfloor .
\end{aligned}
$$

(ii) Optimal inspection time:

$t_{k}=\frac{c_{1}}{c c_{2}}\left(w-\frac{c k}{c_{1}}\right)\left\{1-\ln \left(w-\frac{c k}{c_{1}}\right)\right\}+\left(T-\frac{c_{1}}{c c_{2}}\right), \quad k=1, \ldots, n_{I}$,

where

$$
w=\exp \left[1+W\left(\frac{c c_{2} T-c_{1}}{e c_{1}}\right)\right]
$$

(iii) Condition that the number of inspections is $m$ :

$$
r_{4}(m) \leq T<r_{4}(m+1)
$$

where

$$
r_{4}(m)=\frac{1}{c c_{2}}\left\{\left(m c+c_{1}\right) \ln \left(\frac{m c}{c_{1}}+1\right)-m c\right\} .
$$

(iv) Condition that inspection is effective:

$$
T \geq r_{4}(1)
$$

3.2. When time-to-failure distribution is unknown. Obtained equations in a closed form are summarized in Table 3.2. When $L(t)=c_{1} t^{p}+c_{2}$ and $a=\lambda(0)$, the following results are derived.

(i) Number of inspections:

$$
\begin{aligned}
n_{I} & =\left\lfloor\int_{0}^{1 / a(p+1)} n_{1}^{* *}(t) d t\right\rfloor \\
& =\left\lfloor\left(\frac{c_{1}}{c(p+1)} \cdot \frac{1}{(a p)^{p}}\right)^{1 /(p+1)}\right\rfloor .
\end{aligned}
$$


Table 3.2. Summary of results obtained when time-to-failure distribution is unknown. $L_{1}: L(t)=$ $c_{1} t^{p}+c_{2}, L_{2}: L(t)=c_{1} t^{2}+c_{2} t+c_{3}, L_{3}: L(t)=c_{1}\left(\exp \left[c_{2} t\right]-1\right)+c_{3} ; C_{1}$ : number of inspections, $C_{2}:$ optimal inspection time, $C_{3}$ : condition that the number of inspections is $m(m=1,2, \ldots), C_{4}$ : condition that inspection is effective; $\bigcirc$ : explicit form solution, $\times$ : no explicit form solution.

\begin{tabular}{l|cccc|cccc|cccc}
\hline Loss rate & \multicolumn{5}{|c|}{$L_{1}$} & \multicolumn{5}{c|}{$L_{2}$} & \multicolumn{5}{c}{$L_{3}$} \\
\hline Classification & $C_{1}$ & $C_{2}$ & $C_{3}$ & $C_{4}$ & $C_{1}$ & $C_{2}$ & $C_{3}$ & $C_{4}$ & $C_{1}$ & $C_{2}$ & $C_{3}$ & $C_{4}$ \\
\hline & $\bigcirc$ & $\bigcirc$ & $\bigcirc$ & $\bigcirc$ & $\times$ & $\times$ & $\times$ & $\times$ & $\times$ & $\times$ & $\times$ & $\times$ \\
\hline
\end{tabular}

(ii) Condition that inspection is effective:

$$
a \leq \frac{1}{p}\left(\frac{c_{1}}{c(p+1)}\right)^{1 / p} .
$$

(iii) Condition that the number of inspections is $m$ :

$$
r_{5}(m+1)<a \leq r_{5}(m)
$$

where

$$
r_{5}(m)=\frac{1}{p}\left(\frac{c_{1}}{c(p+1)}\right)^{1 / p} m^{-(p+1) / p}
$$

(iv) Optimal inspection time:

$$
t_{k}=\frac{1}{a(p+1)}\left[1-\left\{1-k\left\{\frac{c(p+1)}{c_{1}}(a p)^{p}\right\}^{1 /(p+1)}\right\}^{(p+1) / p}\right], \quad k=1, \ldots, n_{I} .
$$

In some actual situations, the maximum life span of an inspected system may be given instead of the initial failure rate. Thus, the condition of $\inf \{t \mid F(t)=1\}=t_{M}$ is considered, from which the following equations are derived:

$$
n_{1}^{* *}(t)=\left(\frac{c_{1} p}{c(p+1)^{2}} \cdot \frac{1}{t_{M}-t}\right)^{1 /(p+1)} \quad \text { for } 0 \leq t<t_{M} .
$$

(i) Number of inspections:

$$
\begin{aligned}
n_{I} & =\left\lfloor\int_{0}^{t_{M}} n_{1}^{* *}(t) d t\right\rfloor \\
& =\left\lfloor\left\{\frac{c_{1}}{c}\left(\frac{t_{M}}{p}\right)^{p}(p+1)^{p-1}\right\}^{1 /(p+1)}\right\rfloor .
\end{aligned}
$$

(ii) Condition that the number of inspections is $m$ :

$$
r_{6}(m+1)<t_{M} \leq r_{6}(m)
$$


Table 4.1. Optimal inspection schedules when time-to-failure distribution is given. c.v.: coefficient of variation.

\begin{tabular}{cll}
\hline Loss rate & c.v. & Optimal inspection time \\
\hline \multirow{2}{*}{$L_{1}(t)$} & $1 / 3$ & $\{11,15,18\}$ \\
& $2 / 3$ & $\{7,13,17\}$ \\
& 1 & $\{5,11,16\}$ \\
\hline \multirow{2}{*}{$L_{2}(t)$} & $1 / 3$ & $\{7,10,13,15,17,19\}$ \\
& $2 / 3$ & $\{4,7,10,13,15,18,20\}$ \\
& 1 & $\{3,6,8,11,14,17,20\}$ \\
\hline \multirow{2}{*}{$L_{3}(t)$} & $1 / 3$ & $\{7,10,12,14,16,18,20\}$ \\
& $2 / 3$ & $\{4,7,9,12,14,17,19\}$ \\
& 1 & $\{3,5,8,10,13,16,18\}$ \\
\hline \multirow{2}{*}{$L_{4}(t)$} & $1 / 3$ & $\{3,6,8,10,11,13,14,15,17,18,19,20\}$ \\
& $2 / 3$ & $\{2,4,6,7,9,11,12,14,15,17,18,20\}$ \\
& 1 & $\{2,3,5,7,8,10,11,13,15,16,18,20\}$ \\
\hline
\end{tabular}

where

$$
r_{6}(m)=p\left(\frac{c}{c_{1}} \cdot \frac{m^{p+1}}{(p+1)^{p-1}}\right)^{1 / p}
$$

(iii) Optimal inspection time:

$$
t_{k}=t_{M}\left[1-\left\{1-k\left\{\frac{c}{c_{1}}\left(\frac{p}{t_{M}}\right)^{p} \cdot \frac{1}{(p+1)^{p-1}}\right\}^{1 /(p+1)}\right\}^{(p+1) / p}\right], \quad k=1, \ldots, n_{I} .
$$

(iv) Condition that inspection is effective:

$$
t_{M} \geq r_{6}(1)
$$

When $L(t)=c_{1} t^{2}+c_{2} t+c_{3}$ and $L(t)=c_{1}\left(\exp \left[c_{2} t\right]-1\right)+c_{3}$, it is difficult to derive results in a closed form.

\section{Numerical examples of a virtual system}

Optimal inspection schedules to a virtual maintained system are discussed. Table 4.1 shows the optimal inspection schedules when density function $f(t)$ is the Weibull distribution (mean 15 [year], coefficient of variation: c.v. $=1 / 3$ and $2 / 3$ ) and a negative exponential distribution (mean 15 [year], c.v. $\equiv 1$ ) on the condition of $c=1, L_{1}(t)=t+c_{2}$, $L_{2}(t)=t^{2}+c_{2}, L_{3}(t)=t^{2}+t+c_{3}$, and $L_{4}(t)=(\exp [2 t]-1) / 2+c_{3}$. The optimal solutions are calculated in the range of $\min \left(20,\left\{t \mid F(t) \leq 1-10^{-6}\right\}\right)$, and rounded to the nearest integer. We can see that the inspection interval shortens with time when c.v. $=1 / 3$, 
Table 4.2. Optimal inspection schedules when time-to-failure distribution is unknown. $I(x)$ satisfies $\inf \{t \mid F(t)=1\}=x$.

\begin{tabular}{|c|c|c|}
\hline Loss rate & Condition & Optimal inspection time \\
\hline \multirow{7}{*}{$L_{1}(t)$} & $\lambda(0)=10^{-2}$ & $\{13\}$ \\
\hline & $\lambda(0)=10^{-3}$ & $\{\varnothing\}$ \\
\hline & $\lambda(0)=10^{-4}$ & $\{\varnothing\}$ \\
\hline & $I(5)$ & $\{3,5\}$ \\
\hline & $I(10)$ & $\{5,9,10\}$ \\
\hline & $I(15)$ & $\{7,11,15\}$ \\
\hline & $I(20)$ & $\{8,14,18,20\}$ \\
\hline \multirow{7}{*}{$L_{2}(t)$} & $\lambda(0)=10^{-2}$ & $\{5,10,15,19\}$ \\
\hline & $\lambda(0)=10^{-3}$ & $\{11\}$ \\
\hline & $\lambda(0)=10^{-4}$ & $\{\varnothing\}$ \\
\hline & $I(5)$ & $\{3,5\}$ \\
\hline & $I(10)$ & $\{3,6,8,10\}$ \\
\hline & $I(15)$ & $\{4,7,10,13,15\}$ \\
\hline & $I(20)$ & $\{4,8,12,15,17,20\}$ \\
\hline \multirow{7}{*}{$L_{3}(t)$} & $\lambda(0)=10^{-2}$ & $\{5,10,14,18\}$ \\
\hline & $\lambda(0)=10^{-3}$ & $\{11\}$ \\
\hline & $\lambda(0)=10^{-4}$ & $\{\varnothing\}$ \\
\hline & $I(5)$ & $\{2,4,5\}$ \\
\hline & $I(10)$ & $\{3,6,8,9,10\}$ \\
\hline & $I(15)$ & $\{4,7,10,12,14,15\}$ \\
\hline & $I(20)$ & $\{4,8,11,14,16,18,20\}$ \\
\hline \multirow{7}{*}{$L_{4}(t)$} & $\lambda(0)=10^{-2}$ & $\{2,5,7,9,11,13,15,16,18,19\}$ \\
\hline & $\lambda(0)=10^{-3}$ & $\{3,7,10,13,16,20\}$ \\
\hline & $\lambda(0)=10^{-4}$ & $\{4,9,13,17\}$ \\
\hline & $I(5)$ & $\{2,3,4,5\}$ \\
\hline & $I(10)$ & $\{2,4,6,7,9,10\}$ \\
\hline & $I(15)$ & $\{2,4,6,8,10,12,13,14,15\}$ \\
\hline & $I(20)$ & $\{2,5,7,9,11,13,15,16,18,19,20\}$ \\
\hline
\end{tabular}

whereas the interval is a constant value when c.v. $=1$ and optimal inspection times are not rounded. The first inspection time becomes earlier in proportion to $L_{i}(t)(i=1,2,3$, and 4).

The optimal inspection schedules when the time-to-failure distribution is unknown are shown in Table 4.2 in the range of $\min \left(20,\left\{t \mid F(t) \leq 1-10^{-6}\right\}\right)$, where (2.28) and (2.30) are numerically solved using LSODE [4]. When the initial failure rate is given, the 
following can be seen in proportion to $L_{i}(t)(i=1,2,3$, and 4$)$ : (1) the number of inspections increases, (2) the first inspection time shortens, and (3) the inspection interval shortens. If the maximum life span of an inspected system is given instead of the initial failure rate, a long duration life and a severe loss rate function cause the need for frequent inspection. If the loss rate function has a mild property as in $L_{1}(t)$, inspection is conducted in the neighborhood of the maximum life span. On the other hand, if the loss rate function has a severe property, the first inspection is carried out at an earlier time.

\section{Conclusions}

Optimal inspection schedules for equipment of a single-unit system were discussed in this paper. The approximate expected cost per cycle is the objective where the loss rate function is treated in a general form. The variational method was used to optimize inspection schedules. Optimization problems were classified from the point of whether failure distribution of a system is given or not. When the time-to-failure distribution is given, optimal inspection densities and inspection schedules were derived in a closed form for some loss rate functions. When the failure distribution is unknown, an ordinary differential equation that the optimal inspection density function satisfies and inspection schedules were derived. Then, numerical examples were shown and characteristics of optimal solutions were discussed.

The objective of the optimization problem in this paper is the expected cost per cycle, in which the functional is nonfractional. If a criterion is the expected incurred cost per unit time over an infinite time horizon, in which the functional is fractional, the optimal inspection schedules may differ. Our future work is to examine the effect of the objectives on optimal schedules.

\section{Acknowledgment}

This work was partially supported by Grant-in-Aid for Scientific Research (KAKENHI) no. 16560716 from JSPS.

\section{References}

[1] R. E. Barlow and F. Proschan, Mathematical Theory of Reliability, SIAM Series in Applied Mathematics, John Wiley \& Sons, New York, 1965.

[2] D. I. Cho and M. Parlar, Survey of maintenance models for multi-unit systems, European Journal of Operational Research 51 (1991), no. 1, 1-23.

[3] M. Giaquinta and S. Hildebrandt, Calculus of Variations. I, 2nd ed., Springer, Berlin, 2004.

[4] A. C. Hindmarsh, ODEPACK, a systematized collection of ODE solvers, Scientific Computing (Montreal, Que, 1982) (and R. S. Stepleman, et al., eds.), IMACS Trans. Sci. Comput., I, IMACS, New Jersey; North-Holland, Amsterdam, 1983, pp. 55-64.

[5] N. Kaio and S. Osaki, Some remarks on optimum inspection policies, IEEE Transactions on Reliability 33 (1984), no. 4, 277-279.

[6] J. B. Keller, Optimum checking schedules for systems subject to random failure, Management Science 21 (1974), no. 3, 256-260.

[7] F. K.-N. Leung, Inspection schedules when the lifetime distribution of a single-unit system is completely unknown, European Journal of Operational Research 132 (2001), no. 1, 106-115. 
[8] S. Okumura and N. Okino, An inspection policy for a stochastically failing single-unit system, Proceedings of EcoDesign2003: Third International Symposium on Environmentally Conscious Design and Inverse Manufacturing (Tokyo, 2003), IEEE Computer Society Press, 2003, pp. $137-$ 140.

[9] W. P. Pierskalla and J. A. Voelker, A survey of maintenance models: the control and surveillance of deteriorating systems, Naval Research Logistics Quarterly 23 (1976), no. 3, 353-388.

[10] B. K. N. Rao, Handbook of Condition Monitoring, Elsevier Science, Oxford, 1996.

[11] Y. S. Sherif and M. L. Smith, Optimal maintenance models for systems subject to failure - a review, Naval Research Logistics Quarterly 28 (1981), no. 1, 47-74.

[12] H. Wang, A survey of maintenance policies of deteriorating systems, European Journal of Operational Research 139 (2002), no. 3, 469-489.

Susumu Okumura: Department of Mechanical Systems Engineering, University of Shiga Prefecture, Hikone, Shiga 522-8533, Japan

E-mail address: okumura@mech.usp.ac.jp 


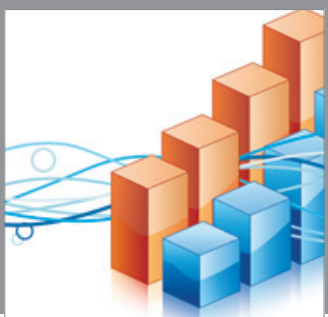

Advances in

Operations Research

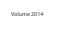

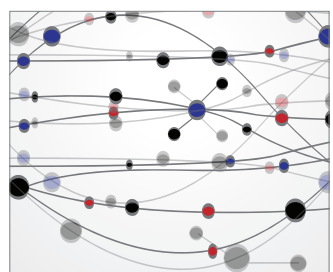

\section{The Scientific} World Journal
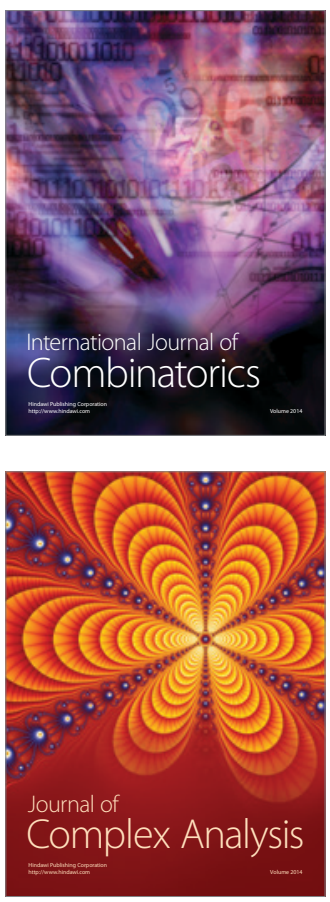

International Journal of

Mathematics and

Mathematical

Sciences
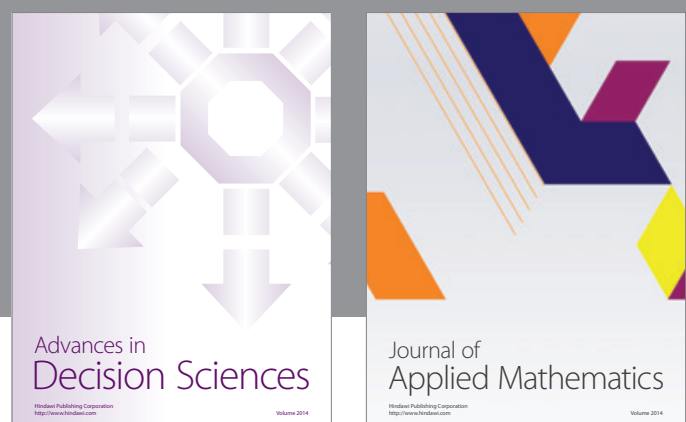

Journal of

Applied Mathematics
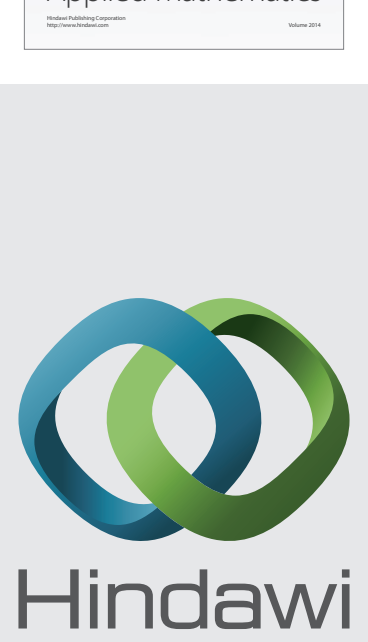

Submit your manuscripts at http://www.hindawi.com
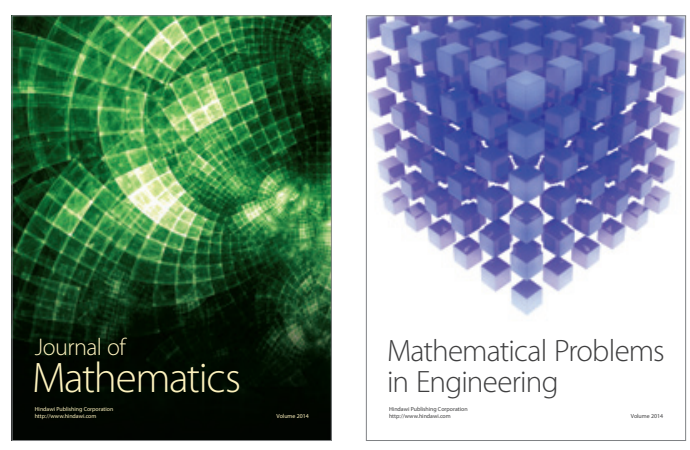

Mathematical Problems in Engineering
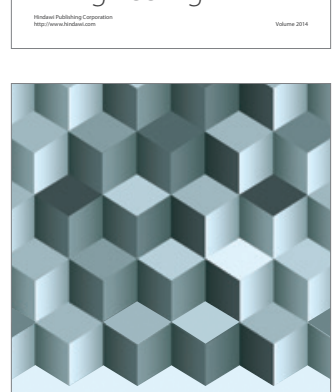

Journal of

Function Spaces
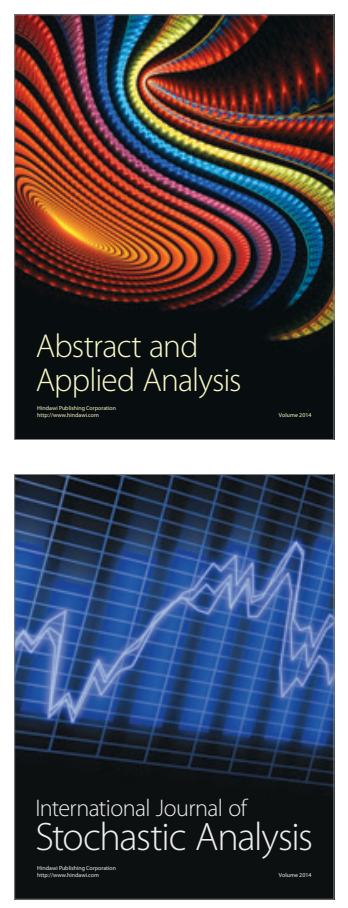

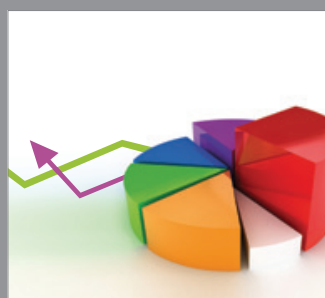

ournal of

Probability and Statistics

Promensencen
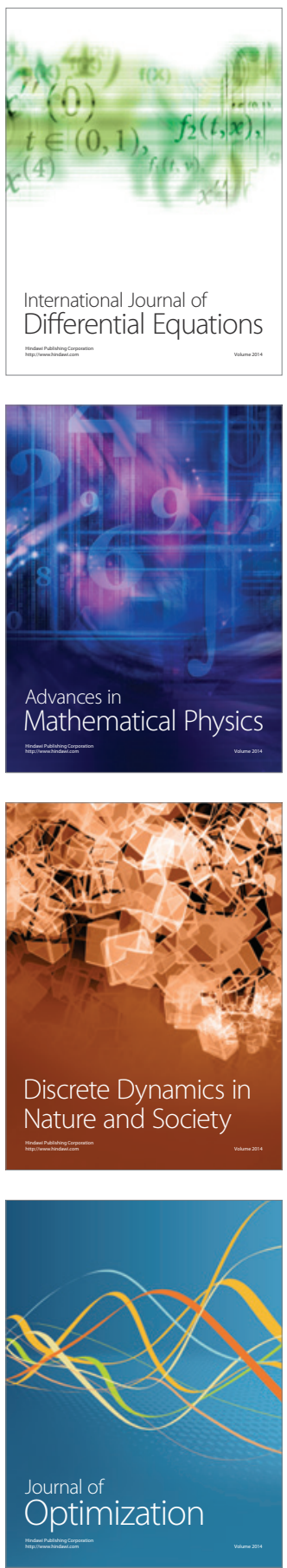